\title{
Assessment, typo error on Author's response of Post Ischemic Stroke Infections
}

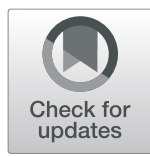

\author{
Mohammad AL-Bahy M. Gamal Reda', Ashraf EL-Sherbiny Abd EL-Hady², Hassan Kawashty Gad', \\ Mohammad Abd EL-Hady M. Aboelmakarem", Amir Abdel-Ghaffar Mohammady Nowar ${ }^{1}$ and \\ Haytham Khalifa Hegazy ${ }^{1,2}$
}

\begin{abstract}
Background: Infection is a common complication in acute phase after stroke, and even in specialized stroke units, it remains one of the major complications. The relationship between infections and acute ischemic stroke is complex and bidirectional. Infections can result in ischemic stroke, and the rate of infection is increased after ischemic stroke.

Aim of work: The aim of this study is to estimate the incidence of post-stroke infections in the Egyptian patients, with determination of the most common infections, possible risk factors, and their clinical implications.

Methods: The study was conducted on one hundred adult patients with acute ischemic stroke, full medical history, thorough clinical examination, routine laboratory investigations, estimation of erythrocyte sedimentation rate and C-reactive protein, abdominal ultrasound, plain X-ray of the chest, culture and sensitivity, non-contrast computed tomography, magnetic resonance imaging for patients with normal CT studies of the brain, duplex study of both carotids and vertebral, ECG, and echocardiography, any further investigations needed and lastly collected data statistically analyzed.

Results: The results of our study showed that 32 patients (32\%) developed post-stroke infection. Post-stroke infections increased with higher age and female gender. Post-stroke infections were strongly correlated to the presence of atrial fibrillation, previous stroke, or transient ischemic attacks. The occurrence of post-stroke infection was significantly correlated to stroke severity, size of infarction, and carotid stenosis. Post-stroke infection was also correlated with the occurrence of multiple post-stroke neurological complications. Infections that occurred poststroke were pneumonia (21\%) and urinary tract infection (15\%), blood (5\%), and subcutaneous infection (3\%).

Conclusion: Stroke is a major health problem, and infection is one of the commonest post-stroke complications. Post-stroke infection significantly affects clinical outcome and being strongly associated with occurrence of neurological complications.

Abstract: study was conducted on one hundred adult patients with acute ischemic stroke, full medical history, thorough clinical examination, routine laboratory investigations, estimation of erythrocyte sedimentation rate and C-reactive protein, abdominal ultrasound, plain X-ray of the chest, culture and sensitivity, non-contrast computed tomography (CT), magnetic resonance imaging (MRI) for patients with normal CT studies of the brain, duplex study of both carotids and vertebral, electrocardiography (ECG), and echocardiography, any further investigations needed and lastly collected data statistically analyzed.
\end{abstract}

Keywords: Stroke, Infection, Risk factor, Pneumonia

\footnotetext{
* Correspondence: Mohamedhady900@yahoo.com

${ }^{1}$ Al-Azhar University, Cairo, Egypt

Full list of author information is available at the end of the article
}

\section{Springer Open}

(c) The Author(s). 2020 Open Access This article is licensed under a Creative Commons Attribution 4.0 International License, which permits use, sharing, adaptation, distribution and reproduction in any medium or format, as long as you give appropriate credit to the original author(s) and the source, provide a link to the Creative Commons licence, and indicate if changes were made. The images or other third party material in this article are included in the article's Creative Commons licence, unless indicated otherwise in a credit line to the material. If material is not included in the article's Creative Commons licence and your intended use is not permitted by statutory regulation or exceeds the permitted use, you will need to obtain permission directly from the copyright holder. To view a copy of this licence, visit http://creativecommons.org/licenses/by/4.0/. 


\section{Background}

Infection is a common complication in acute phase after stroke (Vermeij et al. 2009), and even in specialized stroke units, it remains one of the major complications (Meisel et al. 2005). The relationship between infections and acute ischemic stroke is complex and bidirectional. Infections can result in ischemic stroke, and the rate of infection is increased after ischemic stroke (Ionita et al. 2011). Infection rates after stroke were ranging from 5 to $65 \%$ in some studies (Vermeij et al. 2009), and from 21 to $65 \%$ in other studies (Westendorp et al. 2011). The incidence of infection among stroke patients is thus significantly higher than in general population, prevalence of hospital-acquired infection, which ranges from 6 to 9\% in all hospitalized patients (Anderson 2013). Bacterial pneumonia and urinary tract infection (UTI) are the predominant infections in acute stroke patients (Hilker et al. 2003). Tables $1 \mathrm{~b}$ and 2 showed risk factor of poststroke pneumonia and common pathogen responsible for post-ischemic stroke pneumonia. About one-third of all stroke patients develop infections mostly involving the respiratory and the urinary tract (Vargas et al. 2006); Table 3 showed different type of infections in our study design. Post-stroke infections were typically explained as a result of neurological deficits such as impaired protective reflexes, dysphagia, and decreased consciousness, or as treatment associated complications related to mechanical ventilation or indwelling urinary catheters (Ersoz et al. 2007); Tables 4 and 5 showed risk factors of postischemic stroke UTI and pathogen responsible for UTI. More recent evidence suggests that brain injury itself induces a disturbance of the normally well balanced brainimmune interactions resulting in an immune depressive status with subsequent infections (Dirnagl et al. 2007).

\section{Aim of work}

The aim of this study is to estimate the incidence of post-stroke infections in the Egyptian patients, with

Table 1 Risk factors for post-ischemic stroke pneumonia

\begin{tabular}{lll}
\hline & HR & $p$ \\
\hline Bulbar symptoms & 23 & $<0.001$ \\
Ryle & 316 & $<0.001$ \\
Female gender & 6.4 & 0.001 \\
Age $>$ 61.5 & 19.1 & $<0.001$ \\
AF & 6.5 & $<0.001$ \\
Previous CVS or TIA & 31 & 0.001 \\
NIHSS $>9.5$ & 58.7 & $<0.001$ \\
Size $>$ small & 7.5 & $<0.001$ \\
Carotid stenosis $>27.5$ & 2.4 & 0.093 \\
Catheter & 3.8 & 0.009 \\
MV & 8.9 & 0.999 \\
\hline
\end{tabular}

Table 2 Pathogens responsible for post-ischemic stroke pneumonia in our study patients

\begin{tabular}{ll}
\hline Organism & Number of affected patients \\
\hline Staph. (coagulase-ve) & 5 \\
MRSA & 5 \\
Pseudomonas & 4 \\
E. coli & 4 \\
Acinetobacter & 3 \\
Klebsiella & 2 \\
Candida & 1 \\
\hline
\end{tabular}

determination of the most common infections, possible risk factors, and their clinical implications.

\section{Patients and methods}

The study was conducted on one hundred adult patients with acute ischemic stroke. Inclusion criteria: patient with recent onset of ischemic stroke (less than $48 \mathrm{~h}$ from onset of symptoms), evident by clinical examination and radiological imaging, age more than 18 years. Exclusion criteria: patient with other types of stroke rather than ischemic stroke, patient taking cytotoxic or immunesuppressive drugs, patient with known autoimmune diseases, cancer, hematological disease, and severe renal or liver diseases. All patients were subjected to the following: full medical history, thorough clinical examination including neurological examination, and assessment of the patient's score according to National Institutes of Health Stroke Scale (NIHSS). Routine laboratory investigations include the following: (complete blood count $(\mathrm{CBC}), \mathrm{BUN}$, creatinine, uric acid, sodium $(\mathrm{Na})$, potassium $(\mathrm{K})$, fasting blood sugar (FBS), postprandial blood sugar (PPBS), glycosylated hemoglobin (HbA1c), aspartate transaminase (AST), alanine transaminase (ALT), prothrombin time (PT), prothrombin concentration $(\mathrm{Pc})$, international normalization rate (INR), lipid profile, and urine analysis. Erythrocyte sedimentation rate (ESR) and C-reactive protein (CRP), abdominal ultrasound, plain X-ray of the chest, culture and sensitivity $(C \& S)$, non-contrast computed tomography (CT) study of the brain, magnetic resonance imaging (MRI) of the brain done for patients with normal CT studies, duplex study of both carotids and vertebral,

Table 3 Different types of infections in study patients

\begin{tabular}{ll}
\hline Type of infection & $(n=32), n(\%)$ \\
\hline Chest & 21 patients, (66\%) \\
UTI & 15 patients, (47\%) \\
Blood & 5 patients, (15\%) \\
Subcutaneous and bed sores & 3 patients, (9\%) \\
CNS & 0 \\
\hline
\end{tabular}


Table 4 Risk factors for post-ischemic stroke UTI

\begin{tabular}{lll}
\hline & HR & $p$ \\
\hline Catheter & 36 & $<0.001$ \\
NIHSS $>$ 9.5 & 17.52 & $<0.001$ \\
Female gender & 5 & 0.01 \\
Age $>$ 61.5 & 5.3 & 0.014 \\
AF & 5.3 & 0.005 \\
Previous CVS or TIA & 6.97 & 0.014 \\
Size $>$ small & 27.03 & 0.002 \\
Duplex $>$ 27.5 & 7.6 & 0.011 \\
Bulbar & 0.573 & 0.488 \\
Ryle & 2.02 & 0.255 \\
\hline
\end{tabular}

electrocardiography (ECG), and echocardiography of the heart. Any further investigation is needed to confirm the presence of an infection in a suspected patient as cerebrospinal fluid analysis (CSF), CT chest, CT abdomen. Statistical analysis of collected data by statistical package for the social science (SPSS) version 21.

\section{Results}

The study included 100 patients who were hospitalized because of having recent stroke. Eighty-four patients (84\%) of them were males and 16 patients (16\%) were females. The mean age of all patients was $61 \pm 10$ years. Post-stroke infections occurred in 32 patients (32\%). The patients were divided into 2 groups, (group A) including 68 patients who did not have post-stroke infection, and (group B) including 32 patients who had poststroke infections. The mean hospital stay for all patients was $12.6 \pm 11.7$ days. The mean hospital stay for patients who had post-stroke infections was $22.9 \pm 16$ days, whereas the mean hospital stay for patients who did not have infections was $7.7 \pm 3$ days. It was found that patients who had infection were older in age, have more females, and stayed in the hospital longer than those who did not have infection. No significant differences were found between the two groups regarding the presence of ischemic heart disease (IHD), rheumatic fever, or peripheral vascular disease (PVD). On the other hand, patients who had infections showed significantly more history of previous cerebral vascular stroke (CVS) and previous transient ischemic attacks (TIAs). Interestingly, patients

Table 5 Pathogens responsible for UTI in our study patients

\begin{tabular}{ll}
\hline Organism & Number of affected patients \\
\hline E. coli & 12 \\
Klebsiella & 2 \\
Acinetobacter & 1 \\
Proteus V. & 1 \\
Candida & 1 \\
\hline
\end{tabular}

with infection also had significantly higher rate of atrial fibrillation (AF). Patients who had infection had higher ESR, lower hemoglobin levels, higher total leucocytic count, while they did not have significant difference in platelet counts. Table 6 showed laboratory values of our study design. Patients with infection showed worse renal functions as evident by BUN and creatinine level, while the liver enzymes AST and ALT were not different between the 2 groups. Fasting blood sugar, two-hour postprandial blood sugar, and $\mathrm{HbA} 1 \mathrm{C} \%$ were nearly similar between both groups. Regarding the lipid profile, the total triglycerides and cholesterol levels were not different between both groups, while the low-density lipoprotein (LDL) - cholesterol-was higher in the infection group. Finally, compared to patients who did not have infections, blood electrolytes sodium, potassium, uric acid levels, and the clotting variables (PT and INR) were all insignificantly different from patients who had infections. Comparing the results of 2 groups, there was no significant difference regarding type of affected circulation (whether anterior or posterior), or the affected artery (anterior cerebral artery (ACA), middle cerebral artery (MCA), posterior cerebral artery (PCA), or vertebrobasilar system). However, patients with infection had significantly higher NIHSS score, more occurrence of bulbar symptom, brain edema, and more deterioration in level of consciousness. Table 7 showed stroke state and occurrence of neurological

Table 6 Laboratory values of our study patients

\begin{tabular}{llll}
\hline & Group A (68) & Group B (32) & $p$ value \\
\hline ESR & $21.5 \pm 10.4$ & $79.9 \pm 19.7$ & $<0.001$ \\
HGB & $13.9 \pm 2$ & $12.5 \pm 1.8$ & 0.001 \\
TLC & $6.6 \pm 1.7$ & $12.9 \pm 5.7$ & $<0.001$ \\
PLT & $245.8 \pm 70$ & $231.8 \pm 57$ & 0.327 \\
AST & $22.1 \pm 11$ & $25.2 \pm 9.5$ & 0.166 \\
ALT & $23.2 \pm 15.8$ & $23.4 \pm 11$ & 0.937 \\
BUN & $16.4 \pm 5.2$ & $22.8 \pm 8$ & $<0.001$ \\
Cr & $1.07 \pm 0.42$ & $1.36 \pm 0.66$ & 0.012 \\
FBS & $141.4 \pm 50.8$ & $140 \pm 42.3$ & 0.901 \\
PPBS & $186.3 \pm 59$ & $204.5 \pm 52.4$ & 0.139 \\
HBA1C & $8.12 \pm 2.3$ & $8.5 \pm 2.2$ & 0.436 \\
TG & $198.7 \pm 147$ & $171.8 \pm 66.9$ & 0.329 \\
Cholesterol & $205.3 \pm 48$ & $215.1 \pm 59$ & 0.397 \\
LDL & $130 \pm 36$ & $150 \pm 49$ & 0.026 \\
Na & $134.2 \pm 22.8$ & $138.3 \pm 4.2$ & 0.334 \\
K & $4.1 \pm 1$ & $3.7 \pm 0.51$ & 0.077 \\
UA & $5.86 \pm 1.8$ & $5.64 \pm 1.6$ & 0.552 \\
PC & $78 \pm 27$ & $76 \pm 19$ & 0.690 \\
INR & $1.16 \pm 0.36$ & $1.19 \pm 0.18$ & 0.639 \\
\hline
\end{tabular}


Table 7 Regarding stroke status and occurrence of neurological complications in study patients

\begin{tabular}{llll}
\hline & Group A (68) & Group B (32) & $p$ value \\
\hline NIHSS & $7.19 \pm 3.1$ & $14.2 \pm 3.7$ & $<0.001$ \\
Size (S/M/L) & $50 / 17 / 2$ & $7 / 9 / 15$ & $<0.001$ \\
Circulation (ant/post/both) & $55 / 13 / 1$ & $23 / 6 / 2$ & 0.425 \\
Artery & & & 0.064 \\
$\quad$ ACA & 7 & 0 & \\
MCA & 48 & 26 & \\
PCA & 8 & 0 & \\
VB & 7 & 7 & $<0.001$ \\
Bulbar & 6 & 14 & $<0.001$ \\
B edema & 0 & 12 & $<0.001$ \\
DCL & 2 & 12 & 0.003 \\
Recurrence & 0 & 4 & 0.006 \\
Carotid stenosis by duplex & $29.1 \pm 18$ & $41.3 \pm 21$ & \\
\hline
\end{tabular}

complications. Interestingly, patients with infection had significantly larger size of infarction as assessed by neuro-imaging. Table 8 showed prevalence of predictors of infections in our study. Moreover, significant correlation was found between occurrence of infection and the rate of reduction of arterial flow in the carotid arteries as assessed by duplex studies.

\section{Discussion}

The present study showed that infection commonly complicates acute phase after stroke. In our study, 32 patients developed infection in the acute phase poststroke which implies to an infection rate of $32 \%$. This rate was similar to the infection rate reported in the meta-analysis by Westendorp et al. (2011). By comparing this rate of infection occurring post-stroke to the average rate of nosocomial infections in our hospital (5\%), we found that stroke significantly increased the rate of nosocomial infections. This was in agreement with Meisel et al. (2005) who reported post-stroke infection rate ranging from 21 to $65 \%$, while the average nosocomial infection rate was ranging from 6 to $9 \%$ in all hospitalized patients. We found that the most common infections were pneumonia (66\% of infections and $21 \%$

Table 8 Prevalence of predictors of infection in our study patient

\begin{tabular}{llll}
\hline & Group A (68) & Group B (32) & $p$ value \\
\hline Age $>$ 61 & 20(29\%) & $28(87 \%)$ & $<0.001$ \\
NHISS $>9.5$ & 12 & 31 & $<0.001$ \\
Size $>$ small & 18 & 25 & $<0.001$ \\
Duplex $>$ 27.5 & 21 & 21 & 0.001 \\
BUN $>18.5$ & 26 & 23 & 0.002 \\
\hline
\end{tabular}

of all patients), and the second most common was urinary tract infections ( $47 \%$ of infections and $15 \%$ of all patients). The other reported infections were blood infection and subcutaneous infection with markedly lower incidence rates (5\% and $3 \%$ of all patients) respectively. And interestingly, no central nervous system (CNS) infection was recorded in any patient in our study. These results are similar to that reported by Westendorp et al. (2011), who also found pneumonia and UTI to be the 2 most common post-stroke infections. However, Westendorp et al. (2011), in his metaanalysis stated that there was a wide range of variations in the infection rate of pneumonia (1-33\%) and urinary tract infections (UTI) (2-27\%). On the other hand, Harms et al. (2011) found that UTI occurred more common than pneumonia with an infection rate ranging from 6 to 27\%, while that of pneumonia was ranging from 5 to $22 \%$. A very significant correlation was found between the severity of stroke as indicated by a higher NIHSS score and the development of infection ( $p$ value $<0.001)$. This is completely in agreement with Minnerup et al. (2010) who also found that higher NIHSS score was associated with all types of infections. We also found a significant correlation between the size of the infarction and the development of infection ( $p$ value < $0.001)$. In our study, lesion was divided according to the size: to small $(<1.5 \mathrm{~cm})$, moderate $(1.5-5 \mathrm{~cm})$, and large infarctions ( $>5.0 \mathrm{~cm}$ or $>1 / 3$ of the MCA territory). In our study, a significant association was noticed between the occurrence of post-stroke neurological complications (brain edema, disturbed conscious level (DCL), and stroke recurrence) and the occurrence of infections. Interestingly, we also found a significant association between the rate of reduction in blood flow in carotid arteries elicited by carotid duplex and the development of infections. Aslanyan et al. (2004) also found that poststroke infection rate was associated with the patients' clinical condition. Studies including patients with a higher stroke severity or lower levels of consciousness showed higher infection rates, in particular for pneumonia. This effect corresponds with previous studies that often report both characteristics as risk factors for pneumonia. We found that patients who developed infections are significantly older in age $(68.8 \pm 10.2)$, with higher female predominance (34\%), comparing to those who did not have stroke $57.8 \pm 7.8$ and $7 \%$ respectively. This was in agreement with Ovbiagele et al. (2006) who also found that advanced age was a risk factor for post-stroke infection. We also found a significant correlation between the occurrence of post-stroke infection and a positive history for stroke or transient ischemic attack or both of them with a $p$ value of $<0.001$ for each of them. On the contrary, no association could be found between infections and each of diabetes mellitus (DM), 
hypertension (HTN), and hyperlipidemia, IHD, or PVD. And interestingly, a significant correlation was found between the presence of atrial fibrillation and the development of post-stroke infection ( $p$ value $=0.001)$. We also found that, the best predictors of post-stroke infections are higher age $>61$ years, higher NIHSS score $>9.5$, infarction size that is larger than small and more severe carotid stenotic lesions causing $>27.5 \%$ flow reduction as elicited by duplex study. In our study, pneumonia was the commonest post-stroke infection occurring to 21 patients (21\%). Risk factors significantly associated with post-stroke pneumonia were bulbar manifestations, Ryle insertion, age $>61$, female gender, AF, NIHSS $>9.5$, size of infarction larger than small and previous CVS or TIA. Westendorp et al. (2011) found that pneumonia is the most common post-stroke infection, and that most strokes related pneumonias are believed to result from dysphagia and subsequent aspiration of oropharyngeal material or gastric content. In our study, the commonest organisms responsible for post-stroke pneumonia were coagulase negative Staph (5 patients), methicillin resistant staphelococcus aureus (MRSA) (5 patients), Pseudomonas (4 patients), E-coli (4 patients), Acinetobacter (3 patients), Klebsiella (2 patients), and Candida (1 patient). Gram-negative bacteria and Staphylococcus aureus are known to cause pneumonia by aspiration of endogenous material from the colonized oropharynx (Millns et al. 2003). These pathogens are often seen in nosocomial infections. On the other hand, Streptococcus species is still the most detected pathogen in community acquired pneumonia (Jones 2010). Our results regarding the commonest pathogens were almost in agreement with Westendorp et al. (2011) who found that microbiologic data of patients with post-stroke pneumonia shows a pattern of mostly early onset nosocomial pneumonia, or a community acquired aspiration syndrome. Staphylococcus aureus and gram-negative bacteria such as Klebsiella pneumoniae, Pseudomonas aeruginosa, Escherichia coli, or Enterobacter were commonly identified, also Streptococcus species are occasionally found. Poststroke pneumonia usually occurs within the first 3 to 5 days after hospitalization and thus can be considered as early-onset (HAP) hospital-acquired pneumonia (Schwarz et al. 2008). Harms et al. (2011) stated that early-onset HAP is primarily attributed to Gramnegative bacteria, such as Haemophilus influenzae, and Gram-positive bacteria such as methicillin (methicillin)sensitive Staphylococcus aureus (MSSA) and S. pneumoniae, while late-onset nosocomial pneumonia is usually attributed to higher-level antibiotic-resistant Gramnegative bacteria (e.g., Pseudomonas aeruginosa, Acinetobacter spp.) and Gram-positive bacteria (e.g., MRSA). In our study, urinary tract infection was the second most common post-stroke infection that affected 15 of our patients (15\%). The most important factor associated with post-stroke UTI was insertion of urinary catheter ( $p$ value $<0.001)$. Other significantly associated risk factors were female gender, old age, AF, NIHSS > 9.5, size of infarction larger than small, and previous CVS or TIA. Stott et al. (2009) similarly reported that a higher age and female sex were found to be significant risk factors for urinary tract infection. In our study, different pathogens isolated from urine cultures were E. coli (12 patients), Klebsiella (2 patients), Acinetobacter (1 patient), Proteus V. (1 patient), and candida (1 patient). Zhanel et al. (2005) found that the most common pathogen in UTI remains Escherichia coli (55-80\%). In about $5-10 \%$ of cases, other Entero-bacteriaceae, such as Proteus mirabilis and Klebsiella spp., can be isolated, and occasionally, Staphylococcus saprophyticus is isolated.

\section{Conclusion}

Stroke is a major health problem, and infection is one of the commonest complications following it. Risk factors for post-stroke infections are higher age, female gender, atrial fibrillation, and previous CVS or TIA. Post-stroke infection is strongly associated with larger size of infarction as elicited by neuroimaging. Similarly, it is strongly associated with greater carotid stenosis. Most common types of infections occurring in the post-stroke phase are pneumonia (21\%) and urinary tract infection (15\%). In addition to the abovementioned risk factors, the presence of bulbar symptoms and insertion of Ryle markedly increases the risk of post-stroke pneumonia, while insertion of Foley's urinary catheter markedly increases the risk of post-stroke UTI. Post-stroke infection significantly affects clinical outcome through increasing the length of hospital stay after stroke and being strongly associated with occurrence of neurological complications.

\section{Abbreviations \\ AF: Atrial fibrillation; ACA: Anterior cerebral artery; AST: Aspartate transaminase; ALT: Alanine transaminase; CBC: Complete blood count; CNS: Central nervous system; CVS: Cerebrovascular stroke; CRP: C-reactive protein; CT: Computed tomography; C\&S: Culture and sensitivity; DCL: Disturbed conscious level; ECG: Electrocardiography; ESR: Erythrocyte sedimentation rate; FBS: Fasting blood sugar; HAP: Hospital-acquired pneumonia; HbA1c: Glycosylated hemoglobin; HTN: Hypertension; INR: International normalized ratio; IHD: Ischemic heart disease; LDL: Low density lipoprotein; MCA: Middle cerebral artery; MRI: Magnetic resonance image; MRSA: Methecillin resistant staphylococcus aureus; Na: Sodium; NIHSS: National institute of health stroke scale; K: Potassium; PCA: Posterior cerebral artery; PC: Prothrombin concentration; PT: Prothrombin time; PPBs: Postprandial blood sugar; PVD: Peripheral vascular disease; SPSS: Statistical package for social science; TIAs: Transient ischemic attacks}

\section{Acknowledgements}

I want to express my genuine gratitude to all my professors and colleagues at the Department of Neurology, Al-Azhar University for their consideration and constant support that help me endure hardships.

\section{Authors' contributions}

Professor/ Mohammad AL-Bahy M. is a general supervisor for all research. Professor/ ashraf elsherbiny abdelhady responsible for clinical general 
evaluation of patients. Professor/ Hassan kawashty gad responsible for clinical neurological evaluation of patients. Assistant professor/ Mohammad abdelhady aboelmakarem responsible for statistical analysis of result. Assistant professor/amir abdelgaffar mohammady responsible for editing and writing of discussion. Resident/ haytham khalifa hegazy responsible for contact with patient and collecting data from patients. The author(s) read and approved the final manuscript.

\section{Funding}

no financial grand

\section{Availability of data and materials}

All data generated or analyzed during this study are included in this published article [and its supplementary information files].

\section{Ethics approval and consent to participate}

We confirm that we have read the Journal's position on issues involved in ethical publication and affirm that this report is consistent with those guidelines. This paper approved from Al-Azhar ethical committee.

\section{Consent for publication}

All patients participate in this study informed and consented for this study, and publication was done.

\section{Competing interests}

The authors declare that they have no competing interests.

\section{Author details}

${ }^{1}$ Al-Azhar University, Cairo, Egypt. ${ }^{2}$ Internal Medicine, National Research Center, Giza, Egypt.

Received: 31 May 2019 Accepted: 23 March 2020

Published online: 15 April 2020

\section{References}

Anderson P. Medical management still bests intracranial stenting. Medscape Medical News [serial online]. October 31, 2013; Accessed November 5, 2013. Available at http://www.medscape.com/viewarticle/813604.

Aslanyan S, Weir CJ, Diener HC, Kaste M, Lees KR (2004) Pneumonia and urinary tract infection after acute ischaemic stroke: a tertiary analysis of the GAIN International trial. Eur J Neurol 11:49-53

Dirnagl U, Klehmet J, Braun JS et al (2007) Stroke-induced immunodepression: experimental evidence and clinical relevance. Stroke 38(2 Suppl):770-773

Ersoz M, Ulusoy H, Oktar MA et al (2007) Urinary tract infection and bacteriuria in stroke patients: frequencies, pathogen microorganisms, and risk factors. Am J Phys Med Rehabil 86(9):734-741

Harms H, Halle E, Meisel A (2011) Post-stroke infections - diagnosis, prediction, prevention and treatment to improve patient outcomes. European neurological review:39-43

Hilker R, Poetter C, Findeisen N et al (2003) Nosocomial pneumonia after acute stroke: implications for neurological intensive care medicine. Stroke 34:975-981

Ionita CC, Siddiqui AH, Levy El, Hopkins LN, Snyder KV, Gibbons KJ (2011 Jan-Feb) Acute ischemic stroke and infections. J Stroke Cerebrovasc Dis. 20(1):1-9

Jones RN (2010) Microbial etiologies of hospital-acquired bacterial pneumonia and ventilator-associated bacterial pneumonia. Clin Infect Dis 51(Suppl 1): S81-S87

Meisel C, Schwab JM, Prass K, Meisel A, Dirnagl U (2005) Central nervous system injury-induced immune deficiency syndrome. Nat Rev Neurosci 6(10):775-786

Millns B, Gosney M, Jack Cl, Martin MV, Wright AE (2003) Acute stroke predisposes to oral gram-negative bacilli - a cause of aspiration pneumonia? Gerontology 49:173-176

Minnerup J, Wersching H, Brokinkel B, Dziewas R, Heuschmann PU, Nabavi DG, Ringelstein EB, Schäbitz WR, Ritter MA (2010) The impact of lesion location and lesion size on poststroke infection frequency. J Neurol Neurosurg Psychiatry. 81(2):198-202

Ovbiagele B, Hills NK, Saver JL, Johnston SC (2006) Frequency and determinants of pneumonia and urinary tract infection during stroke hospitalization. J Stroke Cerebrovasc Dis 15:209-213
Schwarz S, Al-Shajlawi F, Sick C, Meairs S, Hennerici MG (2008) Effects of prophylactic antibiotic therapy with mezlocillin plus sulbactam on the incidence and height of fever after severe acute ischemic stroke: the Mannheim infection in stroke study (MISS). Stroke 39(4):1220-1227

Stott DJ, Falconer A, Miller H, Tilston JC, Langhorne P (2009) Urinary tract infection after stroke. QJM 102:243-249

Vargas M, Horcajada JP, Obach V et al (2006) Clinical consequences of infection in patients with acute stroke: is it prime time for further antibiotic trials? Stroke 37(2):461-465

Vermeij FH, Scholte op Reimer WJ, de Man P et al (2009) Stroke-associated infection is an independent risk factor for poor outcome after acute ischemic stroke: Data from the Netherlands Stroke Survey. Cerebrovasc Dis 27:465-471

Westendorp WF, Nederkoorn PJ, Vermeij JD, Dijkgraaf MG, van de Beek D (2011) Post-stroke infection: a systematic review and meta-analysis. BMC Neurol 11

Zhanel GG, Hisanaga TL, Laing NM, DeCorby MR, Nichol KA (2005) Antibiotic resistance in outpatient urinary isolates: final results from the North American Urinary Tract Infection Collaborative Alliance (NAUTICA). Int J Antimicrob Agents 26(5):380-388

\section{Publisher's Note}

Springer Nature remains neutral with regard to jurisdictional claims in published maps and institutional affiliations.

\section{Submit your manuscript to a SpringerOpen ${ }^{\circ}$ journal and benefit from:}

- Convenient online submission

- Rigorous peer review

- Open access: articles freely available online

- High visibility within the field

- Retaining the copyright to your article

Submit your next manuscript at $\boldsymbol{\nabla}$ springeropen.com 